\title{
Thomas Mann
}

\section{Das Recht auf Zugang zu Dienstleistungen von allgemeinem wirtschaftlichem Interesse}

Daseinsvorsorge im EG-Vertrag; Dienstleistungen von allgemeinem wirtschaftlichem Interesse; Europäische Grundrechte-Charta; Förderung des sozialen und territorialen Zusammenhalts

Die Dienstleistungen von allgemeinem wirtschaftichem Interesse werden bereits in Art. 16 und 86 Abs. 2 des EG-Vertrages thematisiert, doch erst Art. 36 der Europäischen Grundrechte-Charta (GRC) statuiert ein Recht auf Zugang zu diesen Dienstleistungen. Gemessen an den Rechtsfindungskriterien des Europäischen Gerichtshofs kann dieses Recht jedoch nicht als Grundrecht der Europäischen Union gewertet werden. Zu Recht verweist Art. 36 GRC deshalb lediglich auf die einzelnen Rechtsordnungen der Mitgliedstaaten und erkennt insoweit bestehende Traditionen bezüglich des Zugangs zu solchen Dienstleistungen an. Durch die Aufnahme der GRC in den Entwurf einer Europäischen Verfassung ist es nun aber vorstellbar geworden, dass das auf französich-romanischer Tradition zurückgehende Recht des Zugangs zu Dienstleistungen von allgemeinem wirtschaftlichem Interesse bald europaweit anzuerkennen ist.

\section{Einführung}

Dienstleistungen von allgemeinem wirtschaftlichem Interesse dienen grundsätzlich der Sicherung von Lebensbedürfnissen der Bürger. Dementsprechend werden diese Dienstleistungen durch Art. 16 und 86 Abs. 2 des EG-Vertrages (EGV) sowie durch Art. 36 der Grundrechte-Charta (GRC) als bedeutendes Element bei der Förderung des sozialen und territorialen Zusammenhalts herausgestellt. Im Unterschied zu den Vorschriften des EGVertrages postuliert Art. 36 GRC aber erstmals ein Recht auf Zugang zu Dienstleistungen von allgemeinem wirtschaftlichem Interesse. Ein Vorbild für dieses subjektive Recht findet sich allerdings weder in der Europäischen Menschenrechtskonvention, noch lässt es sich auf eine gemeinsame Verfassungsüberlieferung der Mitgliedstaaten zurückführen. Dieses Zugangsrecht erfüllt damit nicht die Anforderungen, nach denen die Rechtsprechung des Europäischen Gerichtshofs (EuGH) europäische Grundrechte identifiziert. Der Konvent, der die GRC erarbeitet hat, hat also nicht ein bereits vorhandenes europäisches Grundrecht „sichtbar“ gemacht, wie es sein Auftrag gewesen ist, sondern er hat insoweit nur auf einfachrechtliche Traditionslinien in einigen Mitgliedstaaten verwiesen. Diese Annahme sieht sich bestätigt in der Erläuterung des Präsidiums zu Art. 36 GRC, die betont, dass der Artikel „voll“ Art. 16 EGV achte und „nicht ein neues Recht“ begründe, sondern lediglich den „Grundsatz“ aufstelle, dass die Union den Zugang zu den Dienst- 
leistungen von allgemeinem wirtschaftlichem Interesse gemäß den einzelstaatlichen Bestimmungen achte. ${ }^{1}$

\section{Politische und rechtliche Entwicklung}

\section{Allgemeine politische Entwicklung}

Während in den Anfangsjahren der Europäischen Gemeinschaft auf politischer Ebene die wirtschaftliche Integration und damit die Ausräumung von Handelsbarrieren zwischen den Mitgliedstaaten im Mittelpunkt stand, verlagerte sich die Aufmerksamkeit ab Mitte der achtziger Jahre durch die Liberalisierungsbemühungen in den Bereichen Telekommunikation, Postdienste, Verkehr und Energie auf Sektoren, in denen primär Dienstleistungen von allgemeinem wirtschaftlichem Interesse erbracht werden. Problematisiert wurde v.a. die janusköpfige Rolle des Staates, der einerseits das Funktionieren der Marktmechanismen sicherstellen und andererseits das öffentliche Interesse gewährleisten, insbesondere für die Befriedigung der Grundbedürfnisse der Bürger und die Erhaltung von Kollektivgütern sorgen soll, wenn der Markt dazu nicht in der Lage ist. Zu diesem Spannungsfeld hat die Kommission mehrere Stellungnahmen veröffentlicht. Den Anfang markiert eine Mitteilung aus dem Jahr 1996, in der sie beschrieb, welche Wechselwirkungen zum Wohle der Bürger zwischen den Gemeinschaftsmaßnahmen in den Bereichen Wettbewerb und freier Warenverkehr einerseits sowie den Aufgaben bei der Erbringung öffentlicher Dienstleistungen andererseits bestehen, und vorschlug, die Förderung der Dienstleistungen von allgemeinem Interesse als zusätzliches Vertragsziel aufzunehmen. $^{2}$ Nach einer Aktualisierung dieses Dokuments im Jahre 2000, die dem Ziel dienen sollte, den Akteuren größere Rechtssicherheit bei der Anwendung der Wettbewerbs- und Binnenmarktregeln auf ihre Tätigkeit zu geben, ${ }^{3}$ thematisierte die Kommission zudem in einem Bericht für den Europäischen Rat in Laeken aus dem Jahr 2001 die Problematik der wirtschaftlichen Lebensfähigkeit der mit der Erbringung öffentlicher Dienstleistungen betrauten Akteure und die Verantwortung der Mitgliedstaaten bei der Festlegung von Gemeinwohlzielen. ${ }^{4}$ Den vorläufigen Abschluss bildet ein Grünbuch aus dem Jahre 2003, mit dem die Kommission u.a. die Diskussion über die Möglichkeiten und Grenzen einer Rechtsetzung der Gemeinschaft im Bereich der Dienstleistungen von allgemeinem Interesse anstoßen möchte. ${ }^{5}$

Vgl. CHARTE 4473/00, Convent 49, S. 28.

2 Vgl. Kommission, Leistungen der Daseinsvorsorge in Europa, ABl. EG Nr. C 281 v. 26. September 1996, S. $3 \mathrm{ff}$.

3 Vgl. Kommission, Leistungen der Daseinsvorsorge in Europa, ABl. EG Nr. C 17 v. 19. Januar 2001, S. 4 ff.

4 Vgl. Kommission, Bericht für den Europäischen Rat in Laeken „Leistungen der Daseinsvorsorge“, KOM (2001) 598, vgl. dazu Kämmerer (2002), S. 1041 ff.

5 Vgl. Kommission, „Grünbuch zu Dienstleistungen von allgemeinem Interesse“, KOM (2003) 270; s. dazu Ruge (2003), S. 353 ff. 


\section{Vorgaben des Primärrechts}

Die Dienstleistungen von allgemeinem wirtschaftlichem Interesse werden im primären Gemeinschaftsrecht in den Art. 16 EGV und Art. 86 Abs. 2 EGV thematisiert. Art. 86 EGV, die ältere dieser beiden Vorschriften ${ }^{6}$, legt in Abs. 1 fest, dass die Bestimmungen des Vertrags, insbesondere das Diskriminierungsverbot (Art. 12 EGV) und die Wettbewerbsregeln (Art. 81 - 89 EGV), prinzipiell auch auf öffentliche Unternehmen Anwendung finden. Dieser vor dem Hintergrund des europäischen Wettbewerbsmodells konsequente Grundsatz wird in Absatz 2 Satz 1 ,,für Unternehmen, die mit Dienstleistungen von allgemeinem wirtschaftlichem Interesse betraut sind oder den Charakter eines Finanzmonopols haben“ relativiert, ${ }^{7}$,,soweit die Anwendung dieser Vorschriften nicht die Erfüllung der ihnen übertragenen besonderen Aufgaben rechtlich oder tatsächlich verhindert". Die Vorschrift entfaltet innerstaatliche Wirksamkeit in dem Sinne, dass sie unmittelbar anwendbar ist und von den mit der Erbringung von Dienstleistungen Betrauten vor nationalen Behörden und Gerichten für sich reklamiert werden kann. ${ }^{8}$ Für diese gelten also die Vertragsbestimmungen - insbesondere die Wettbewerbsregeln der Art. 81 ff. EGV - ausnahmsweise dann nicht, wenn deren Anwendung die Erfüllung einer dem Erbringer der Dienstleistung übertragenen besonderen Aufgabe ,rechtlich oder tatsächlich verhindert". Jede Abweichung von den Spielregeln des Wettbewerbs steht also unter Rechtfertigungszwang. In Anbetracht der bei Legalausnahmen wie Art. 86 Abs. 2 S. 1, 2. Hs. EGV grundsätzlich gebotenen restriktiven Interpretation ${ }^{9}$ hat die EuGH-Rechtsprechung klargestellt, dass eine bloße Behinderung oder Erschwerung nicht ausreichend ist, ${ }^{10}$ sondern die Anwendung des Vertrags mit den Aufgaben des Unternehmens ,nachweislich unvereinbar" sein muss. ${ }^{11}$

6 Der Grundsatz des Art. 16 EGV wurde erst durch den Amsterdamer Vertrag v. 2. Oktober 1997 (BGBl. II 1999, S. 296) eingefügt.

7 Der thematische Zusammenhang der beiden Absätze, der insbesondere durch die verklammernde Aufgabenzuweisung („Anwendung dieses Artikels“) an die Kommission in Art. 86 Abs. 3 EGV bestätigt wird, lässt vermuten, dass mit den in Abs. 2 umschriebenen Unternehmen keine neue, von Abs. 1 grundverschiedene Unternehmenskategorie angesprochen sein soll, sondern dass es sich hierbei zumindest um eine Teilmenge der öffentlichen Unternehmen i.S.d. Abs. 1 handelt - vgl. auch EuGH v. 19. Mai 1993, Rs. 320/91, Slg. I 1993, 2533 Rn. 13 - Corbeau: Abs. 1 sei „in Verbindung mit Abs. 2 zu lesen“. Allgemein zu der zwischen Bindung (Abs. 1), Freistellung (Abs. 2) und Rückbindung (Abs. 3) oszillierenden Tatbestandsstruktur des Art. 86 EGV Mann (2002b), S. 823.

8 EuGH v. 11. April 1989, Rs. 66/86, Slg. 1989, 803 Rn. 57 f. - Ahmed Saeed; EuGH v. 18. Juni 1991, Rs. C260/89, Slg. I 1991, 2925 Rn. 38 - ERT; EuGH v. 27. April 1994, Rs. C-393/92, Slg. I 1994, 1477 Rn. 51 Almelo; EuGH v. 19. Oktober 1995, Rs. C-19/93 P, Slg. I 1995, 3319 Rn. 9 - Rendo; die Rechtsprechung zusammenfassend Generalanwalt Darmon, Schlussantrag v. 8. Februar 1994, Rs. C-393/92, Slg. 1994, I-1477 (Rn. 124) - Almelo.

9 EuGH v. 27. März 1974, Rs. 127/73, Slg. 1974, 313 (Rn. 19/22) - BRT II; Emmerich ( 1998), H. II Rn. 144; Kahl (1996), S. 1083; Pielow (2000), S. 78; Dohms (2001), S. 57; Cox (2002), S. 332; Kämmerer (2004), S. 31.

10 EuGH v. 20. März 1985, Rs. 41/83, Slg. 1985, 873 Rn. 33 - British Telecom; EuGH v. 10. Dezember 1991, Rs. C-179/90, Slg. 1991, I-5889 Rn. 26 f. - Porto di Genova; EuG v. 16. Juni 1997, Rs. T-260/94, Slg. 1997, II-997 Rn. 138 - Air Inter; etwas abweichend EuGH v. 23. Oktober 1997, Rs. 157/94, Slg. 1997, I-5699 Rn. 43 Kommission/Niederlande: Berufung auf Art. 90 II (heute: Art. 86 II) EGV erfordere nur, dass die Anwendung des Vertrags die Erfüllung der auferlegten Verpflichtungen rechtlich oder tatsächlich ,gefährden“ würde.

11 Vgl. EuGH v. 30. April 1974, Rs. 155/73, Slg. 1974, 409 Rn. 15 - Sacchi; EuGH v. 23. April 1991, Rs. C-41/90, Slg. 1991, I-1979 Rn. 24 - Höfner u. Elser; EuGH v. 18. Juni 1991, Rs. C-260/89, Slg. 1991, I-2925 Rn. 33 - ERT; Generalanwalt Darmon, Schlussantrag v. 8. Februar 1994, Rs. C-393/92, Slg. 1994, I-1477 (Rn. 143). 
Noch bis zur Modifikation durch die Corbeau-Rechtsprechung im Jahr 1993 hatte die Rechtsprechung aufgrund dieser Vorgaben keinen Anwendungsfall der Vorschrift bejaht, sondern stets dem Wettbewerb einen Vorrang eingeräumt. ${ }^{12}$ Seitdem aber konkretisiert der EuGH seine Forderungen an die „tatsächliche“ Verhinderung nach nachweisbarer Unvereinbarkeit von Vertragsanwendung und Aufgabenerfüllung, indem er maßgeblich auf wirtschaftliche Gesichtspunkte abstellt und danach fragt, ob es dem Unternehmen noch möglich ist, seine im Allgemeininteresse liegende Aufgabe unter wirtschaftlich tragbaren Bedingungen zu erfüllen. ${ }^{13}$ Dieser Ansatz erscheint sachgerecht, wenn man bedenkt, dass den Erbringern der Dienstleistungen von allgemeinem wirtschaftlichem Interesse durch die Betrauung mit Gemeinwohlaufgaben gerade die Pflicht aufgegeben ist, Dienstleistungen entgegen ökonomischer ratio auch in unrentablen Sektoren zu erbringen (sog. Universaldienstleistungspflicht), und sie eine wirtschaftlich ausgewogene Geschäftstätigkeit zumeist nur erreichen, indem sie die in den unrentablen Bereichen entstandenen Verluste mit den in anderen Sektoren erwirtschafteten Gewinnen verrechnen. Wenn Wettbewerber nun die wirtschaftlich attraktiven Dienstleistungen erbringen, ohne unrentable Bereiche bedienen zu müssen, können sie ihre Leistungen zu besseren Konditionen anbieten und den mit allgemeinen Dienstleistungen betrauten Unternehmen erhebliche Marktanteile wegnehmen. Letztlich würde somit die Erfüllung dieser im Allgemeininteresse liegenden Aufgabe wirtschaftlich untragbar werden. ${ }^{14}$

Als rechtliche Gründe im Sinne des Art. 86 Abs. 2 EGV, die eine Erfüllung der besonderen, den Unternehmen übertragenen Aufgabe zu verhindern geeignet sind, müssen v.a. die ausdrücklichen Freistellungen angesehen werden, die der Vertrag selbst enthält. ${ }^{15}$ In diesem Kontext ist auch der durch den Amsterdamer Vertrag eingeführte Art. 16 EGV zu beachten, der den Grundsatz akzentuiert, dass in der europäischen Rechtsordnung das Wettbewerbsprinzip nicht uneingeschränkt Geltung beansprucht, sondern dass zugleich auch eine Rücksichtnahme auf die Erfüllung gemeinwohlorientierter Daseinsvorsorgeaufgaben geboten ist. ${ }^{16}$ Er akzentuiert mithin einerseits den Stellenwert der Dienste von allgemeinem wirtschaftlichem Interesse, macht aber andererseits deutlich, dass die Europäische Gemeinschaft die Sonderrolle, die öffentlichen Unternehmen aufgrund ihrer spe-

12 Vgl. z.B. kategorisch EuGH v. 10. Juli 1984, Rs. 72/83, Slg. 1984, 2727 Rn. 19 - Campus Oil. Ein konziser Überblick über die EuGH-Rechtsprechung zu Art. 86 II EGV findet sich bei Götz (2001), S. 925 ff.

13 EuGH v. 19. Mai 1993, Rs. C-320/91, Slg. 1993, I-2533 Rn. 15 f. - Corbeau; EuGH v. 27. April 1994, Rs. C393/92, Slg. 1994, I-1477 Rn. 49 - Almelo; EuGH v. 23. Oktober 1997, Rs. C-157/94, Slg. 1997, I-5699 Rn. 53 - Kommission/Niederlande; EuGH v. 23. Oktber 1997, Rs. C-158/94, Slg. 1997, I-5789 Rn. 53 f. - Kommission/Italien; EuGH v. 23. Oktober 1997, Rs. C-159/94, Slg. 1997, I-5815 Rn. 96 - Kommission/Frankreich; EuGH v. 13. Mai 2003, Rs. C-463/00, Slg. 2003, I-4581 Rn. 82 - Golden Share.

14 Vgl. näher Pielow (2000), S. 87 f.; Frenz (2000), S. 907 ff.

15 Hinzuweisen ist insoweit insbesondere auf Art. 30 und 46 EGV, die jeweils an den Vorbehalt der öffentlichen Ordnung, Sicherheit oder Gesundheit anknüpfen.

16 Die Bedeutung des Art. 16 EGV wird unterschiedlich bewertet; als Ausdruck eines Gleichordnungsverhältnisses von Wettbewerb und staatlichen Dienstleistungen insbes. Schwarze (2001), S. 13 f., 22; in der Tendenz einer Aufwertung der Dienstleistungen ähnlich v.d. Groeben/Schwarze-Hochbaum/Klotz, 6. Aufl., BadenBaden, Art. 86 EGV Rn. 61; Frenz (2000), 916 f.; Pielow (2000), S. 100 f.; Calliess/Ruffert-Jung, EGV, 2. Aufl., Art. 16 EGV Rn. 13; Art. 86 EGV Rn. 49; v. Danwitz (2002), S. 136 = S. 82 f.; Storr (2002), S. 361; Kämmerer (2004), S. 29; als Bekräftigung eines weiterhin bestehenden Regel-Ausnahme-Verhältnisses hingegen Henneke (2000), S. 138; Burgi (2002), S. 265 f.; Kluth (2002), S. 71; Weiß (2003a), S. 130; ders. (2003b), S. 186 
zifischen Funktion als Instrumente zur Durchsetzung wirtschafts- oder sozialpolitischer Ziele der Mitgliedstaaten zukommt, achtet. Im Ergebnis wird durch Art. 16 EGV damit klargestellt, dass ein wirtschaftliches Engagement der öffentlichen Hand auch unter den Bedingungen einer „offenen Marktwirtschaft mit freiem Wettbewerb“ (Art. 4 Abs. 1, 98 S. 2, 105 Abs. 1 S. 3 EGV) zulässig ist, grundsätzlich aber keine Sonderstellung im Hinblick auf die Maßgaben des EGV beanspruchen kann.

\section{Zur Genese des Art. 36 GRC}

Das Recht auf Zugang zu Dienstleistungen von allgemeinem wirtschaftlichem Interesse fand - wenn auch inhaltlich nicht näher spezifiziert - aufgrund einiger Änderungsvorschläge während den Beratungen im Grundrechtskonvent erstmals als Artikelüberschrift zu Art. 45a bzw. 50a der Charta Eingang in einen Kompromissvorschlag des Präsidiums vom 23. Juni 2000. ${ }^{17}$ Erst unmittelbar in der 15. Sitzung des Konvents am 17.-19. Juli 2000 wurde dann kurzfristig und „zur allgemeinen Überraschung “18 die Kopie eines Vorschlags des Präsidiums verteilt, die erstmalig den Text der Bestimmung wie folgt formulierte: „L'accès aux services d'intérêt général est garanti, conformément au traité instituant la Communauté européenne et selon les législations et pratiques nationales“. Die sich hieran entzündende Diskussion verlief sehr konträr, wobei sich als Befürworter der Regelung v.a. Konventsmitglieder aus Frankreich, Luxemburg und Österreich, als Gegner einer Aufnahme dieser Bestimmung in den Text der Charta hingegen vornehmlich Konventsmitglieder aus Deutschland, Großbritannien und den Niederlanden profilierten ${ }^{19}$ - eine Lagerbildung, die sich durchaus vor dem Hintergrund unterschiedlicher nationalstaatlicher Konzepte der Daseinsvorsorge erklären lässt. Während die eine Seite nämlich den sozialen Aspekt eines gleichen und kontinuierlichen Zugangs zur wirtschaftlichen Grundversorgung betonte, ${ }^{20}$ verwies die andere Seite auf die fehlende verfassungsrechtliche Verortung in den nationalstaatlichen Rechtsordnungen und auf unabsehbare Konsequenzen der Anerkennung eines derart neu gestalteten Rechts. ${ }^{21}$ Aufgabe des Grundrechts-Konvents war es nämlich, die Grundrechte abzubilden ,wie sie in der EMRK gewährleistet sind und wie sie sich aus den gemeinsamen Verfassungsüberlieferungen der

17 CHARTE 4373/00, Convent 40, S. 23 und 30.

18 So ausdrücklich das Sitzungsprotokoll, abgedruckt bei Bernsdorf/Borowsky (2002), S. 343.

19 Vgl. das Sitzungsprotokoll bei Bernsdorff/Borowsky (2002), S. 343 ff. sowie die Darstellung bei Meyer-Riedel, Art. 36 GRC Rn. 3 ff.

20 So wurde gefordert, es müsse verhindert werden, dass der Strom abgeschaltet werde, nur weil die Rechnung nicht bezahlt worden sei (Braibant, Frankreich, Protokoll der 15. Sitzung, abgedr. bei Bernsdorff/Borowsky (2000), S. 344 ff.) oder darauf verwiesen, dass der Sinn der Bestimmung darin liege, auch die in entfernten Tälern oder hoch auf dem Berge Wohnenden mit Strom zu versorgen (Einem, Österreich, Protokoll der 16. Sitzung, ebda., S. 371).

21 So v.a. Mombaur, P.M, Deutschland, und Friedrich, I., Deutschland („Büchse der Pandora“), Protokoll der 15. Sitzung, abgedr. bei Bernsdorff/Borowsky (2002), S. 345 sowie Tarschys, Schweden (,Gefahr, dass der EuGH am Ende über jedes Wasserwerk entscheide“), Patijn, Niederlande (es wisse der Konvent ,gar nicht, was er hier garantiere“), Protokoll der 15. Sitzung, ebda., S. 346 und Manzella, Italien (Text sei „zu stark verwässert“), Protokoll der 16. Sitzung, ebda., S. 371. 
Mitgliedstaaten als allgemeine Grundsätze des Gemeinschaftsrechts ergeben“. ${ }^{22}$ Mit der Formulierung eines entsprechenden Rechts auf Zugang zu Dienstleistungen von allgemeinem wirtschaftlichem Interesse in Art. 36 GRC hat der Konvent demzufolge seinen Handlungsauftrag überschritten.

\section{Vorgaben des Sekundärrechts}

Die Vorgaben des Primärrechts werden durch das sogenannte Sekundärrecht (vgl. Art. 249 EGV) sowie sonstige Äußerungen mit Blick auf die beabsichtigte Vorgehensweise der Kommission ergänzt. In Konkretisierung des Art. 86 Abs. 2 EGV regelt das Sekundärrecht bspw. Fälle, in denen Beihilfen zulässig sind. Zwar unterfallen Leistungen der Daseinsvorsorge den regulären Vorgaben der Art. 87 und 88 EGV, doch bilden sie einen Sonderfall des Beihilferechts, was sich daran zeigt, dass diese Leistungen in der Ausnahmenvorschrift des Art. 86 II EGV besonders geregelt sind. Weiterhin haben die Gemeinschaftsorgane für einzelne Bereiche der Daseinsvorsorge sogenannte „Sektorenrichtlinien" erlassen, die auf eine Liberalisierung der Märkte abzielen. Solche Vorgaben sind insbesondere für den Energiesektor, im öffentlichen Personennahverkehr sowie im Telekommunikationssektor relevant. ${ }^{23}$

\section{Zum Gewährleistungsgehalt des Art. 36 GRC}

\section{Zum Terminus „Dienstleistungen von allgemeinem wirtschaftlichem Interesse“}

Die zunächst in Art. 86 Abs. 2 EGV benutzte Bezeichnung „Dienstleistungen von allgemeinem wirtschaftlichem Interesse“, die durch den Vertrag von Amsterdam als „Dienste von allgemeinem wirtschaftlichen Interesse" auch Eingang in Art. 16 EGV gefunden hat, kommt in den Rechtsordnungen der Mitgliedstaaten so nicht vor. Vieles spricht dafür, dass sie, um Art. 86 Abs. 2 EGV einen möglichst weiten Anwendungsbereich zu geben, absichtlich unbestimmt gefasst wurde. ${ }^{24}$

22 Schlussfolgerungen des Europäischen Rates von Köln, Anhang IV vgl. auch den 5. Erwägungsgrund in der Präambel zur Grundrechtscharta, ABl. EG Nr. C 364 v. 18. Dezember 2000, S. 1ff. = CHARTE 4487/00, Convent 50 vom 28. September $2000=$ BR-Dr. 666/00.

23 Vgl. bspw. Richtlinie 90/388/EWG der Kommission vom 28. Juni 1990 über den Wettbewerb auf dem Markt für Telekommunikationsdienste (AB1. EG Nr. L 192 v. 24. Juli 1990, S. 10.), außer Kraft gesetzt durch Art. 10 der Richtlinie 2002/77/EG der Kommission vom 16. September 2002 über den Wettbewerb auf den Märkten für elektronische Kommunikationsnetze und -dienste (ABl. EG Nr. L 249 v. 17. September 2002, S. 21 ff.); Richtlinie 96/92/EG des Europäischen Parlaments und des Rates vom 19. Dezember 1996 betreffend gemeinsame Vorschriften für den Elektrizitätsbinnenmarkt (ABl. EG Nr. L 27 v. 30. Januar 1997, S. 20 ff.), außer Kraft gesetzt durch die Richtlinie 2003/54/EG des Europäischen Parlaments und des Rates vom 26. Juni 2003 über gemeinsame Vorschriften für den Elektrizitätsbinnenmarkt und zur Aufhebung der Richtlinie 96/92/EG (ABl. EG Nr. L 176 v. 15. Juli 2003, S. 37 ff.); Richtlinie 2004/17/EG des Europäischen Parlaments und des Rates vom 31. März 2004 zur Koordinierung der Zuschlagserteilung durch Auftraggeber im Bereich der Wasser-, Energie- und Verkehrsversorgung sowie der Postdienste (ABl. EG Nr. L 134 v. 30. April 2004, S. 114 ff.).

24 Vgl. Generalanwalt Dutheillet de Lamothe, EuGH, Rs. 10/71, Slg. 1971, 723 ff. (739). 


\section{a) Dienstleistung}

Der Begriffsstamm, der Terminus „Dienstleistung“, ist primärrechtlich allerdings in Art. 50 EGV definiert. Danach sind Dienstleistungen im Sinne des Vertrags zunächst einmal schlicht „Leistungen, die in der Regel gegen Entgelt erbracht werden“. Exemplifizierend werden in Art. 50 S. 2 EGV gewerbliche, kaufmännische, handwerkliche und freiberufliche Tätigkeiten aufgelistet. Diese Begriffsbestimmung vermittelt freilich nur Anhaltspunkte für die Auslegung des Rechts auf Zugang zu Dienstleistungen von allgemeinem wirtschaftlichem Interesse, da bereits der Dienstleistungsbegriff innerhalb des Art. 86 Abs. 2 EGV, an dessen Bedeutung sich dieses Recht orientiert, nach überwiegender Ansicht weiter zu fassen ist, weil er etwa auch Sachleistungen erfasst. ${ }^{25}$ Andererseits erfolgt, indem Art. 86 Abs. 2 EGV und daran anschließend auch Art. 36 GRC diesen Terminus durch den Zusatz ,,von allgemeinem wirtschaftlichem Interesse“ ergänzen, eine Eingrenzung dieses recht weiten Regelungsansatzes, die sich alleine aus dem Text des EGV nicht ausreichend erhellen lässt.

\section{b) Begrenzung auf das allgemeine wirtschaftliche Interesse}

Eine - mangels Vertragsauslegungskompetenz der Kommission freilich nicht verbindliche - Definition der „Dienstleistungen von allgemeinem wirtschaftlichem Interesse“ enthält der Anhang II der Mitteilung der Kommission „Leistungen der Daseinsvorsorge in Europa“ aus dem Jahr 2000: ${ }^{26}$ Danach bezeichnet der in Art. 86 EGV verwendete Begriff ,marktbezogene Tätigkeiten, die im Interesse der Allgemeinheit erbracht und daher von den Mitgliedstaaten mit besonderen Gemeinwohlverpflichtungen verbunden werden." In ihrem Grünbuch zu Dienstleistungen von allgemeinem Interesse aus dem Jahr 2003 greift die Kommission diesen Definitionsansatz auf und formuliert sprachlich abweichend, dass diese Formulierung ,sich auf wirtschaftliche Tätigkeiten bezieht, die von den Mitgliedstaaten oder der Gemeinschaft ${ }^{27}$ mit besonderen Gemeinwohlver-pflichtungen verbunden werden und für die das Kriterium gilt, dass sie im Interesse der Allgemeinheit erbracht werden“". ${ }^{28}$ Die Frage, in welchen Wirtschaftssektoren solche Gemeinwohlverpflichtungen anzutreffen sind, beantwortet die Kommission selbst, indem sie exemplifizierend ausführt: „Das Konzept der Dienstleistungen von allgemeinem wirtschaftlichem Interesse umfasst daher insbesondere bestimmte Leistungen der großen netzgebundenen Wirtschaftszweige wie des Verkehrswesens, der Postdienste, des Energiesektors und der Telekommunikation. Der Begriff gilt jedoch auch für jede sonstige wirtschaftliche Tätigkeit, die mit Gemeinwohlverpflichtungen verknüpft ist.“ Hierdurch

25 Vgl. nur Page (1982), S. 28; Emmerich (1998), H II Rn. 148; Pielow (2000), S. 79; Calliess/Ruffert-Jung, 2. Aufl., Art. 86 EGV Rn. 36.

$26 \operatorname{KOM}(2000), 580=$ ABl. EG Nr. C 17 v. 19. Januar 2001, S. 4 ff.

27 Auffallend an dieser neuen Wendung ist, dass die Kommission nun offenbar auch der Gemeinschaft selbst eine Definitionskompetenz für Gemeinwohlziele zuspricht.

28 Vgl. Kommission, Grünbuch zu Dienstleistungen von allgemeinem Interesse, KOM (2003) 270 endg. S. 8 (Rn. 17). 
wird zwar deutlich, in welchen Wirtschaftssektoren solche Gemeinwohl-verpflichtungen anzutreffen sind, doch ist damit nicht ausschließlich das Konzept des service public auf der Gemeinschaftsebene implementiert.

Wenn man den für das Zugangsrecht entscheidenden Begriff in seine Elemente zerlegt, dann stellt die tatbestandliche Verengung der Dienstleistungen auf solche von ,allgemeinem" Interesse klar, dass ausschließlich privatnützige Dienstleistungen nicht Gegenstand des Zugangsrechts sein können. ${ }^{29}$ Es geht mit anderen Worten nur um solche Dienstleistungen, deren Erbringung im öffentlichen Interesse liegt, wobei die Definitionskompetenz über diesen Gemeinwohlbezug nicht auf europäischer Ebene angesiedelt ist, sondern bei den Mitgliedstaaten verbleibt, welche den Dienstleistern daher auch besondere Gemeinwohlverpflichtungen auferlegen können. ${ }^{30}$ Der Begriff der Dienstleistung von allgemeinem Interesse als solcher wird also, wie auch sonst im Europarecht üblich, autonom ausgelegt, nur rekurriert das europarechtliche Verständnis auf eine tatsächlich erfolgte Zuweisung von Gemeinwohlaufgaben an die Unternehmen, die dem Entscheidungsbereich der Mitgliedstaaten überlassen bleibt.

Das entscheidende Merkmal ist aber, dass es sich zudem um Dienstleistungen von „,wirtschaftlichem“ Interesse handeln muss. Damit sind zunächst einmal solche Dienstleistungen aus dem Anwendungsbereich der Art. 16 und 86 Abs. 2 EGV sowie Art. 36 GRC ausgeschlossen, die der Verfolgung kultureller oder sozialer Ziele dienen. ${ }^{31}$ Das Grünbuch der Kommission aus dem Jahr $2003^{32}$ erläutert diese Einschränkung dahingehend, dass sie den Kranz der Dienstleistungen von allgemeinem Interesse auf die marktbezogenen Dienstleistungen reduziert. Um den Anwendungsbereich des Rechts auf Zugang zu Dienstleistungen von allgemeinem wirtschaftlichem Interesse abstecken zu können, ist es mithin notwendig, auf der einen Seite zwischen marktbezogenen und nichtmarktbezogenen Dienstleistungen und auf der anderen Seite zwischen allgemeinen oder partikularen Interessen zu unterscheiden. Soweit sich eine eindeutige Zuordnung innerhalb dieser Begriffspaare nicht treffen lässt, weil eine Dienstleistung z.B. zugleich allgemeinen und partikularen Interessen dient, wird man nicht umhinkommen, als Abgrenzungskriterium auf den Schwerpunkt der Dienstleistung abzuheben. ${ }^{33}$ Im Ergebnis jedenfalls träfe es den Inhalt des Gewollten besser und wäre wohl auch sprachlich präziser, anstelle der Wendung „Dienstleistung von allgemeinem wirtschaftlichem Interesse“ die Formulierung „wirtschaftliche Dienstleistung von allgemeinem Interesse“ zu gebrauchen. ${ }^{34}$

29 EuGH v. 27. März 1974, Rs. 127/73, Slg. 1974, 313 (Rn. 23) - BRT II; Generalanwalt Reischl, Schlussantrag v. 11. Januar 1983, Rs. 7/82, Slg. 1983, 483 (524); Grabitz/Hilf-Pernice/Wernicke, Art. 86 Rn. 37; MeyerRiedel, Art. 36 GRC Rn. 10.

30 EuGH v. 23. Oktober 1997, Rs. C-159/94, Slg. 1997, I-5815 Rn. 56 - Kommission/Frankreich; Alber (2001), S. 108 f.; Frenz (2000), S. 905 f.; Storr (2002), S. 363. Zur Überprüfbarkeit durch den EuGH vgl. EuGH v. 13. Mai 2003, Rs. 463/00, Slg. 2003, I-4581 Rn.70 f. - Golden Share.

31 Tettinger (1994), S. 89; v.d. Groeben/Schwarze-Hochbaum/Klotz, 6. Aufl., Art. 86 EGV Rn. 62; Kahl (1996), S. 1083; Entscheidung 71/224/EWG der Kommission v. 2. Juni 1971, ABl. EG Nr. L 134 v. 20. Juni 1971, S. 15 (27) - GEMA; Mitteilung der Kommission „Leistungen der Daseinsvorsorge in Europa“, KOM (2000), 580, S. 13 , Rn. 28 f.

32 Vgl. Kommission, Grünbuch zu Dienstleistungen von allgemeinem Interesse, KOM (2003) 270, S. 7 (Rn. 16).

33 So der Vorschlag von Emmerich (1998), H II Rn. 152; ihm folgend Meyer-Riedel, Art. 36 GRC Rn. 10.

34 Zutreffend Götz (2001), S. 923. 


\section{Das Recht auf Zugang zu den Dienstleistungen von allgemeinem wirtschaftlichem Interesse}

Soweit Art. 36 GRC nun von der Anerkennung und der Achtung des Zugangs zu den solchermaßen abgesteckten Dienstleistungen spricht, kann damit nicht ein Recht auf Erbringung dieser Dienstleistungen, die nach der Konzeption des Art. 86 Abs. 2 EGV von einer staatlichen Betrauung abhängt, ${ }^{35}$ gemeint sein, sondern nur ein Recht darauf, in den Genuss der angebotenen Dienstleistung zu kommen. Vor diesem Hintergrund stellt sich auch die im Grundrechtekonvent kontrovers diskutierte Frage ${ }^{36}$, ob das Zugangsrecht auch gegenüber privaten Dienstleistern bestehen könne, da es grundsätzlich möglich ist, dass die im Interesse des Gemeinwohls liegenden Dienstleistungen von Privaten erbracht werden. $^{37}$

Art. 36 GRC gewährt in diesen Fällen selbst kein Zugangsrecht, sondern verweist hinsichtlich der Reichweite dieses Zugangsrechts lediglich auf die mitgliedstaatlichen Rechtsordnungen und statuiert deren Achtung und Anerkennung, so dass die „einzelstaatlichen Rechtsvorschriften und Gepflogenheiten“ über den Zugang zu diesen Dienstleistungen durch die Union anerkannt und geachtet werden. Auf diese Weise kann der unterschiedlichen Ausgangslage in den Mitgliedstaaten Rechnung getragen werden, ohne dass durch die Vorschrift - worauf die Erläuterung des Sekretariats des Konventspräsidiums besonderen Wert legt ${ }^{38}$ - ein „neues Recht“ geschaffen würde. ${ }^{39}$ In der Genese des Art. 36 GRC hat sich die Rekursivformel mehrfach verändert. Während es zunächst nur hieß, dass die Union den Zugang zu Dienstleistungen von allgemeinem wirtschaftlichem Interesse ,achtet“ ${ }^{40}$ ist zu einem späteren Zeitpunkt hieraus ein ,anerkennt und achtet“ geworden. ${ }^{41}$ Damit wurde zum einen eine terminologische Vereinheitlichung innerhalb

35 Vgl. dazu EuGH v. 27. März 1974, Rs. 127/73, Slg. 1974, 313 (Rn. 23); EuGH v. 14. Juli 1981, Rs. 172/80, Slg. 1981, 2021 Rn. 7 - Züchner; EuGH v. 11. April 1989, Rs. 66/86, Slg. 1989, 803 Rn. 55 - Ahmed Saeed; EuGH v. 17. Juli 1997, Rs. C-242/95, Slg. 1997, I-4449 Rn. 50 - GT-Link; EuGH v. 23. Oktober 1997, Rs. C159/94, Slg. 1997, I-5815 Rn. 53 - Kommission/Frankreich; EuG v. 27. Februar 1997, Rs. T-106/95, Slg. 1997, II-229 Rn. 173 - FFSA; EuG v. 19. Juli 1997, Rs. T-260/94, Slg. 1997, II-997 Rn. 135 - Air Inter. Die Anforderungen an den Betrauungsakt werden aber nicht überdehnt, vgl. die Kommission, Entscheidung 91/50/EWG v. 16. Januar 1991, ABl. EG Nr. L 28 v. 2. Februar 1991, 32 (43); Entscheidung 93/126/EWG vom 22. Dezember 1992, ABl. EG Nr. L 50 v. 2. März 1993, S. 14 (22), Rn. 28 - Jahrhundertvertrag und Ehlers (1990), S. 1095. Angesichts des in Deutschland definitionsgemäß bestehenden Einflusses der öffentlichen Hand zur Sicherung der öffentlichen Zweckbindung will etwa Burgi (1990), S. 276 bei öffentlichen Unternehmen generell eine Betrauung i.S.d. Art. 86 II EGV annehmen. Ähnlich Badura (1998), S. 823 mit Blick auf kommunale Unternehmen. Umfassender Überblick über den Meinungsstand bei Tettinger (1994), S. 89 f. und Pielow (2000), S. $83 \mathrm{ff}$.

36 Vgl. einerseits Azevedo (Portugal), Protokoll der 15. Sitzung, abgedr. bei Bernsdorff/Borowsky (2002), S. 345 und andererseits Tarschys (Schweden) und Friedrich (Deutschland), ebda., S. 346.

37 Das wird auch von EuGH v. 13. Mai 2003, Rs. 463/00, Slg. 2003, I-4581 Rn. 66 - Golden Share, vorausgesetzt.

38 CHARTE 4473/00, Convent 49 vom 11. Oktober 2000 zu Art. 36 GRC.

39 Gegen dieses Regelungskonzept und für eine positive Zusicherung eines Mindestmaßes an Dienstleistungen für alle Europäer durch die Charta selbst Manzella A.E., Protokoll der 16. Sitzung des Grundrechtskonvents, abgedr. bei Bernsdorff/Borowsky (2002), S. 371.

40 CHARTE 4422/00, Convent 45, S. 11 (Art. 34).

41 Änderung durch CHARTE 4470/00, Convent 47, S. 12 (Art. 35). Die von deutscher Seite zwischenzeitlich vorgeschlagene Formulierung „respektiert“ - vgl. den Hinweis auf einen entsprechenden Formulierungsvorschlag im Protokoll der 16. Sitzung des Grundrechtskonvents, abgedr. bei Bernsdorff/Borowsky (2002), S. 346 - hat sich hingegen nicht durchsetzen können. 
der Charta herbeigeführt (vgl. Art. 25, 26, 34 Abs. 1 GRC), aber auch ein neuer Aussagegehalt hinzugefügt: Eine Anerkennung geht über ein bloßes Achten hinaus, weil mit dieser Wendung deutlich gemacht wird, dass die Union den Rechtsvorschriften und Gepflogenheiten in den Mitgliedstaaten nicht nur passive Aufmerksamkeit und Wertschätzung entgegenbringt, sondern sie auch aktiv gutheißt und bestätigt. ${ }^{42}$

3. Die Bedeutung der gemeinwohlorientierten Dienstleistungen für die Union

Die Erbringung gemeinwohlorientierter Dienstleistungen gehört zum „Kern des Europäischen Gesellschaftsmodells". ${ }^{43}$ Dies zeigt sich in Art. 36 GRC, der in seinem letzten Halbsatz die Zielsetzung zum Ausdruck bringt, dass die Anerkennung und Achtung des Zugangs zu Dienstleistungen von allgemeinem wirtschaftlichem Interesse erfolgt, ,um den sozialen und territorialen Zusammenhang der Union zu fördern" und somit eine Formulierung aufgreift, die sich bereits in Art. 16 EGV findet (,,in Anbetracht ... ihrer Bedeutung bei der Förderung des sozialen und territorialen Zusammenhalts"). Demzufolge ist die Erbringung gemeinwohlorientierter Dienstleistungen im Rahmen der Gemeinschaftspolitik nach Art. 3 Abs. 1 lit k) EGV zu verorten ${ }^{44}$, denn auch nach Ansicht der Kommission trägt die Achtung der nationalstaatlichen Besonderheiten in diesem Bereich dazu bei, die Bindung der Bürger an die EU zu stärken. ${ }^{45}$

\section{Zusammenfassende Bewertung und Ausblick}

Gemeinwohlorientierte Dienstleistungen sind bedeutende Elemente für die Förderung des sozialen und territorialen Zusammenhalts. Während durch Art. 86 Abs. 2 EGV diese Dienstleistungen bereits sehr früh im europäischen Primärrecht thematisiert wurden, erfolgte die Formulierung des Rechts auf Zugang zu Dienstleistungen erst durch den Grundrechte-Konvent. Hierbei handelt es sich jedoch um kein zuvor bereits bestehendes Grundrecht der Europäischen Union und auch die dem Art. 36 GRC zugeordnete ,amtliche“ Erläuterung des Sekretariats des Konventspräsidiums legt Wert auf die Feststellung, dass mit dieser Vorschrift kein neues Recht gegenüber Art. 16 EGV geschaffen wird. ${ }^{46}$ Allerdings muss diese Aussage differenzierter gesehen werden: Die Bestimmung eröffnet zwar keinen eigenständigen Schutzbereich eines europäischen Grundrechts, wie es die „klassischen“ Grundrechte der Charta mit den Formulierungen „Jede Person hat

42 Der in der deutschen Sprachfassung eher marginal erscheinende Unterschied zeigt sich deutlicher in einigen anderen Sprachfassungen, vgl. engl.: „recognises and respects“; frz.: „,reconnaît et respecte“; it.: „,riconosce e rispetta“; span.: „reconoce y respeta“.

43 Vgl. Kommission, Leistungen der Daseinsvorsorge in Europa, ABl. EG Nr. C 281 v. 26. September 1996, C 281/3 Rn. 1; Kommission, Leistungen der Daseinsvorsorge in Europa, KOM (2000) 580, Zusammenfassung, Rn. 7.

44 Calliess/Ruffert-Jung, 2. Aufl., Art. 16 EGV Rn. 11; vgl. aber auch Alber (2001), S. 83, der in dieser Wendung lediglich einen Hinweis auf die Gemeinwohlbezogenheit der Leistungen sieht.

45 Vgl. Kommission, Leistungen der Daseinsvorsorge in Europa, KOM (2000) 580, Rn. 64.

46 CHARTE 4473/00, Convent 49 vom 11. Oktober 2000 zu Art. 36 GRC. 
das Recht ..." zum Ausdruck bringen, doch die Bestimmung rekurriert auf bereits mitgliedstaatlich existierende Zugangsrechte. Bereits dem Wortlaut nach wird damit aber durchaus ein „Mehr“ gegenüber Art. 16 EGV gewährt: Während Art. 16 EGV zunächst einmal nur den Stellenwert und die Bedeutung der Dienste von allgemeinem wirtschaftlichem Interesse achtet, wird in Art. 36 GRC der konkrete „Zugang“ zu diesen Dienstleistungen, so wie er in den Mitgliedstaaten ausgestaltet ist, anerkannt und geachtet, was im Kontext einer Grundrechtssammlung nur als ein Hinweis auf subjektive Rechte auf Teilhabe an den Dienstleistungen verstanden werden kann. Das schon im Vorfeld der Einfügung des Art. 16 EGV in den Vertrag - ohne Erfolg - geforderte subjektive Recht aller EG-Bürger auf Versorgung mit einem Mindeststandard an Leistungen ${ }^{47}$ wird somit gleichsam über den Umweg mitgliedstaatlicher Gewährleistung eingeführt. ${ }^{48}$ Noch beschränkt sich dieses Recht allerdings auf Staaten der französich-romanischen Traditionslinie, die ein solches Zugangsrecht, das sich grundlegend von dem in den deutschen Gemeindeordnungen verankerten Recht der Einwohner auf Zulassung zu kommunalen öffentlichen Einrichtungen unterscheidet, bereits in ihrer mitgliedstaatlichen Rechtsordnung kennen, wie etwa die service public-Konzeption in Frankreich ${ }^{49}$ oder die servicio público-Lehre in Spanien. ${ }^{50}$ Angesichts der Dynamik des Gemeinschaftsrechts dürfte die Prognose allerdings nicht zu gewagt sein, dass mit der Aufnahme eines solchen Zugangsrechts in die Grundrechte-Charta und deren Implementation in die künftige europäische Verfassung auch eine Erstreckung des Zugangsrechts auf die übrigen Mitgliedstaaten nur noch eine Frage der Zeit ist. Ansätze in diese Richtung zeigten sich bereits bei der Diskussion im Grundrechtskonvent und in jüngeren Äußerungen der Kommission. ${ }^{51}$

47 Vgl. Europäisches Parlament, Entschließung zur Rolle des öffentlichen Sektors bei der Vollendung des Binnenmarktes, ABl. EG Nr. C 96 v. 1. April 1996, S. 77 ff.; Europäisches Parlament, Entschließung zu dem allgemeinen Rahmen für einen Entwurf zur Revision der Verträge, ABl. EG Nr. C 33 v. 3. Februar 1997, S. 66 ff.; vgl. auch Rodrigues (1998), S. 41 zu den anfänglichen Plänen der Regierungskonferenz von Turin. Weitergehend de lege lata bereits der Wirtschafts- und Sozialausschuss, Stellungnahme zum Thema „Leistungen der Daseinsvorsorge“" vom 21. Oktober 1999, AB1. EG 1999 Nr. C 368 v. 20. Dezember 1999, S. 51 ff., Ziff. 5.3.

48 Auf mögliche „Wechselwirkungen“ zwischen Art. 16 EGV und Art. 36 GRC hat bereits Kluth (2002), S. 70 hingewiesen.

49 Dazu zuletzt Mann (2005), § 33 Rn. 12 ff.

50 Eingehend hierzu Mann (2002a), S. $327 \mathrm{ff}$.

51 Die Mitteilung der Kommission, Leistungen der Daseinsvorsorge in Europa, AB1. EG Nr. C 17 v. 19 . Januar 2001, S. 4 ff. und das Grünbuch der Kommission zu Dienstleistungen von allgemeinem Interesse aus dem Jahr 2003, KOM (2003) 270 endg., Tz. 63 und Anhang Rn. 23 heben hervor, dass die Garantie des Zuganges für jedermann ein zentrales Element der Verbraucherpolitik im Bereich der Dienstleistungen von allgemeinem wirtschaftlichem Interesse darstelle. Ähnlich zuvor bereits die Kommission, Stellungnahme v. 28. Februar 1996 zum Abschlussbericht der sog. Reflexionsgruppe, KOM (96) 90 endg. = ABl. EG Nr. C 281 v. 26. September 1996, S. 3 = Bulletin der EU 1/2-1996, S. 9 (166): Zu den allen europäischen Gesellschaften gemeinsamen Wertvorstellungen gehöre auch ,der gleichberechtigte Zugang der Bürger zu Universaldiensten sowie zu Versorgungs- und Dienstleistungen, die der solidarischen Daseinsvorsorge dienen“. Vor einer europarechtlichen Begründung von Zugangsrechten warnen daher auch Burgi (2002), S. 271 f. und Storr (2002), S. 361. 
Thomas Mann, Services of general economic interests in the european context

European Charter of Fundamental Rights; Services of general economic interests; support of the social and territorial cohesion

The services of general economic interest have already been a subject of art. 16 and 86 par. 2 EC Treaty but art. 36 Charter of Fundamental Rights stated an access right to these services for the first time. This right cannot however be rated as a fundamental right of the European Union, as art. 36 GRC simply refers to the Member States 'legal systems and only accepts their tradition in regard to such an access. Due to the dynamic nature of the EC Law it is only a matter of time that this access right to services of general economic interest (based on french-roman tradition) will soon be adopted by further Member States.

\section{Literaturverzeichnis}

Alber, Siegbert (2001), Unternehmen der Daseinsvorsorge im europäischen Wettbewerbsrecht, in: Daseinsvorsorge im Lichte des Wettbewerbsrechts, hrsg. von Jürgen Schwarze, Baden-Baden, S. 73-110

Badura, Peter (1998), Wirtschaftliche Betätigung der Gemeinde zur Erledigung von Angelegenheiten der örtlichen Gemeinschaft, in: Die öffentliche Verwaltung, 51. Jg., Heft 19, S. 818-823

Bernsdorff, Norbert und Martin Borowsky (2002), Die Charta der Grundrechte der Europäischen Union, BadenBaden, S. 343

Burgi, Martin (1990), Die öffentlichen Unternehmen im Gefüge des primären Gemeinschaftsrechts, in: Europarecht, 32. Jg., Heft 3, S. 261-290

Burgi, Martin (2002), Verwalten durch öffentliche Unternehmen im europäischen Institutionenwettbewerb, in: Verwaltungsarchiv, 93. Jg., Heft 2, S. 255-277

Cox, Helmut (2002), Dienstleistungen von allgemeinem wirtschaftlichem Interesse in Europa, in: Zeitschrift für öffentlichen und gemeinwirtschaftliche Unternehmen, 25. Jg., Heft 3, S. 331-339

v. Danwitz, Thomas (2002), Dienste von allgemeinem wirtschaftlichem Interesse in der europäischen Wettbewerbsordnung, in: Nordrhein-Westfälische Verwaltungsblätter, 16. Jg., Heft 4, S. 132-139 = Bitburger Gespräche Jahrbuch 2002/I, München, S. 73-89

Calliess, Christian und Matthias Ruffert (2002), Kommentar zu EU-Vertrag und EG-Vertrag, 2. Aufl., Neuwied und Kriftel

Dohms, Rüdiger (2001), Die Vorstellungen der Kommission zur Daseinsvorsorge, in: Daseinsvorsorge im Lichte des Wettbewerbsrechts, hrsg. von Jürgen Schwarze, Baden-Baden, S. 41-72

Ehlers, Dirk (1990), Die wirtschaftliche Betätigung der öffentlichen Hand in der Bundesrepublik Deutschland, in: Juristenzeitung, 45. Jg., Heft 23, S. 1089-1100

Emmerich, Volker (1998), Monopole und öffentliche Unternehmen, in: Handbuch des EU-Wirtschaftsrechts, hrsg. von Manfred A. Dauses, 2. Aufl., München, H. II.

Frenz, Walter (2000), Dienste von allgemeinem wirtschaftlichem Interesse, in: Europarecht, 35. Jg., Heft 6, S. 901925

Götz, Volkmar (2001), Die Betrauung mit Dienstleistungen von allgemeinem wirtschaftlichem Interesse (Art. 86 Abs. 2 EG) als Akt der öffentlichen Gewalt, in: Festschrift für Hartmut Maurer, hrsg. von Max-Emanuel Geis und Dieter Lorenz, München, S. 921-933

v.d. Groeben, Hans und Jürgen Schwarze (2002), Kommentar zum EU/EG-Vertrag, 6. Aufl., Baden-Baden

Henneke, Hans-Günter (2000), Sparkasse-Quo vadis?, in: Niedersächsische Verwaltungsblätter, 7. Jg., Heft 6, S. 129-141

Hochbaum, Ingfried F. und Robert Klotz (2003), in: Kommentar zum EU/EG-Vertrag, hrsg. von Hans v.d. Groeben und Jürgen Schwarze, 6. Aufl., Baden-Baden, Art. 86

Jung, Christian (2002), in: Kommentar zu EU-Vertrag und EG-Vertrag, hrsg. von Christian Calliess und Matthias Ruffert, 2. Aufl., Neuwied und Kriftel, Art. 16 und Art. 86 
Kahl, Wolfgang (1996), Das öffentliche Unternehmen im Gegenwind des europäischen Beihilferegimes, in: Neue Zeitschrift für Verwaltungsrecht, 15. Jg., Heft 11, 1996, S. 1082-1088

Kämmerer, Jörg Axel (2002), Daseinsvorsorge als Gemeinschaftsziel oder: Europas „,soziales Gewissen“, in: Neue Zeitschrift für Verwaltungsrecht, 21. Jg., Heft 9, S. 1041-1045

Kämmerer, Jörg Axel (2004), Strategien zur Daseinsvorsorge, in: Neue Zeitschrift für Verwaltungsrecht, 23. Jg., Heft 1, S. 28-33

Kluth, Winfried (2002), Zur Bedeutung des Art. 16 EGV für die Wahrnehmung von Aufgaben der Daseinsvorsorge durch die Kommunen, in: Kommunale Perspektiven im zusammenwachsenden Europa, hrsg. von Hans-Günter Henneke, Stuttgart u.a., S. 68-87.

Mann, Thomas (2002a), Die öffentlich-rechtliche Gesellschaft, Tübingen

Mann, Thomas (2002b), Öffentliche Unternehmen im Spannungsfeld von öffentlichem Auftrag und Wettbewerb, in: Juristenzeitung, 57. Jg., Heft 17, S. 819-826

Mann, Thomas (2005), Zugang zu Dienstleistungen von allgemeinem wirtschaftlichen Interesse, in: Handbuch des europäischen Grundrechtsschutzes, hrsg. von Carsten Nowak und Sebastian Heselhaus, München, § 33

Page, Alan C. (1982), Member States, Public Undertakings and Article 90, in: European Law Review, 7. Jg., Heft 1, S. $19-35$

Pernice, Ingolf und Stephan Wernicke (2004), in: Kommentar zur Europäischen Union, hrsg. von Eberhard Grabitz und Meinhard Hilf, München, Art. 86

Pielow, Johann-Christian (2000), Grundstrukturen öffentlicher Versorgung, Tübingen

Riedel, Eibe (2003), in: Kommentar zur GRC, hrsg. von Jürgen Meyer, Baden-Baden, Art. 36

Rodrigues, Stéphane (1998), Les services publics et le Traité d'Amsterdam, in: Revue du marché commun et de l'union, 414. Bd., S. 37-46

Ruge, Reinhard (2003), Forum - Das Grünbuch der EG-Kommission zur Leistung der Daseinsvorsorge, in: Zeitschrift für Rechtspolitik, 36. Jg., Heft 10, S. 353-356

Schwarze, Jürgen (2001), Einführung, in: Daseinsvorsorge im Lichte des Wettbewerbs, hrsg. von Jürgen Schwarze, Baden-Baden, S. 9-24

Storr, Stefan (2002), Zwischen überkommener Daseinsvorsorge und Diensten von allgemeinem wirtschaftlichem Interesse, in: Die öffentliche Verwaltung, 55. Jg., Heft 9, S. 357-367

Tettinger, Peter J. (1994), Die öffentlichen Unternehmen im primären Gemeinschaftsrecht, in: Deutsches Verwaltungsblatt, 109. Jg., Heft 2, S. 88-94

Weiß, Wolfgang (2003a), Europarecht und Privatisierung, in: Archiv des öffentlichen Rechts, 128. Jg., Heft 1, S. 91-133

Weiß, Wolfgang (2003b), Öffentliche Unternehmen und EGV, in: Europarecht (EuR), 38. Jg., Heft 2, S. 165-190 